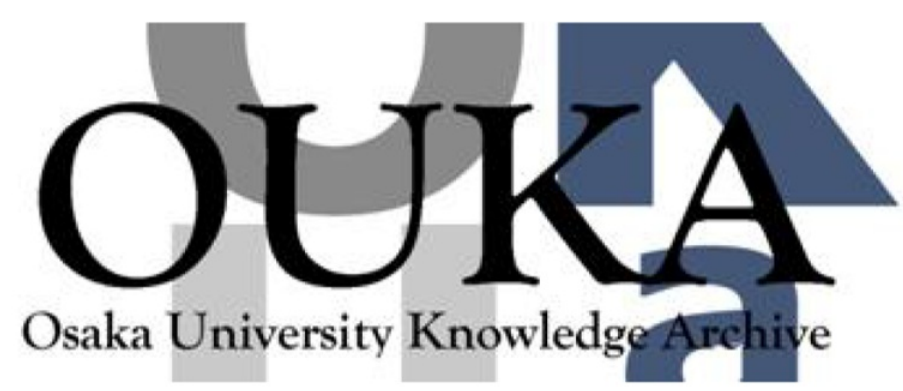

\begin{tabular}{|c|l|}
\hline Title & $\begin{array}{l}\text { Electroabsorption spectroscopy of amorphous } \\
\text { Si/SiC quantum well structures }\end{array}$ \\
\hline Author(s) & Hattori, K.; Tsujishita, M.; Okamoto, H. et al. \\
\hline Citation & Applied Physics Letters. 55(8) p. 763-p. 765 \\
\hline Issue Date & $1989-08-21$ \\
\hline oaire:version & VoR \\
\hline URL & https://hdl. handle. net/11094/3351 \\
\hline rights & \\
\hline Note & \\
\hline
\end{tabular}

Osaka University Knowledge Archive : OUKA

https://ir. Library. osaka-u. ac. jp/

Osaka University 


\title{
Electroabsorption spectroscopy of amorphous Si/Sic quantum well structures
}

\author{
K. Hattori, M. Tsujishita, H. Okamoto, and Y. Hamakawa \\ Faculty of Eingineering Science, Osaka University, Ioyonaka, Osaka 560, Japan
}

(Received 10 April 1989; accepted for publication 9 June 1989)

\begin{abstract}
The interband optical transition in quantum wells of hydrogenated amorphous silicon and silicor carbide has been studied by using electroabsorption (EA) spectroscopy. The observed EA spectrum exhibits a triangular line shape, identified as being due to a field-induced modification of the subband transition. The identification is confirmed by comparing with the experimental result of thermoabsorption spectroscopy.
\end{abstract}

Electroabsorption (EA) in quantum well (QW) structures consisting of alternate ultrathin layers of crystalline semiconductors has recently attracted much interest both for physics and applications. ${ }^{1}$ An electric field perpendicular to the QW layer directly modifies the confined electronic states and corresponding optical transitions. When the potential drop across the layer is small compared to the confinement energy, the optical absorption shifts towards lower photon energy while retaining an abrupt onset. The phenomenon is different in character from a broadening of bandedge absorption due to bulk Franz-Keldysh effect. That is called the quantum-confined Franz-Keldysh (QCFK) effect, ${ }^{2}$ or quantum-confined Stark (QCS) effect especially for the excitonic transitions. ${ }^{1,3,4}$ Similar phenomena could be expected for the amorphous semiconductor $\mathrm{QW}$ structures, in which the quantum confinement effects have been identified experimentally in the differential optical absorption spectrat ${ }^{5,6}$ as well as in the resonant tunneling characteristics.?

In this letter, we report on EA spectrocopy of multiple quantum well (MQW) structures of hydrogenated amorphous silicon $(a-\mathrm{Si}: \mathrm{H})$ and silicon carbide $(a-\mathrm{SiC}: \mathrm{H})$. The measured EA spectrum shows a triangular line shape clearly distinguishable from a broad bump-like shape of bulk EA spectrum. ${ }^{8,9}$ The feature is identified as due to a subband transition in accordance with a simple model of the QCFK effect assuming a nondirect band-to-band transition. ${ }^{10}$ The identification of the EA structure is further supported from the comparison with the thermoabsorption (TA) spectrum measured on the identical MQW structure. Built-in electric fleld in the well layer is estimated from the relation between the EA signal intensity and the applied fieid. Applicability of a square QW model for the confined states in the presence of internal field is discussed.

The structure of samples employed for EA measurement was glass/transparent conductive oxide (TCO) $/ n$ type $a-\mathrm{Si}: \mathrm{H}(400 \AA) /$ undoped $\mathrm{MQW}$ of $a-\mathrm{Si}: \mathrm{H}$ and $a$ $\mathrm{SiC}: H / n$-type $a$-Si:H $(200 \AA) / \mathrm{Al}$. The $a$-Si:H/a-SiC:H MQW structures were prepared by ro plasma chemical vapor deposition with the use of a $1.9 \mathrm{SiH}_{4}-\mathrm{H}_{2}$ gas mixture for $a$ Si:H and I:I 1:108 $\mathrm{SiH}_{4}-\mathrm{CH}_{4}-\mathrm{H}_{2}$ gas mixiure for $a-\mathrm{SiC}: \mathrm{H}$. The $a$-Si:H and $a$-SiC:H layers were formed in separate chambers with an interruption on the plasma. In one of the MQWs, the layer thicknesses of the $a-\mathrm{Si}: \mathrm{H}$ well $\left(L_{W}\right)$ and $a$ $\mathrm{SiC}: \mathrm{H}$ barrier $\left(L_{B}\right)$ were chosen to be 20 and $100 \AA$, respec- tively, and the accumulation number of well layers $(M)$ was 150. As a reference structure having different total thickress, a MQW was prepared with $L_{W}=20 \AA, L_{B}=60 \AA$, and $M=40$. The multiple layered structures were coninrmed to be formed with atomicaily abrupt heterointerfaces from an $x$-ray diffraction measurement." The optical band gaps $\left(E_{0}\right)$ were $1.75 \mathrm{eV}$ for $a-\mathrm{Si}: \mathrm{H}$ and $2.8 \mathrm{eV}$ for $a-\mathrm{SiC}: \mathrm{H}$, respectively. The band discontinuities were estimated to be $0.8 \mathrm{eV}$ for the conduction band and $0.25 \mathrm{eV}$ for the valence band from the results of uitraviolet photoelectron emission measurement.

The electric fied modulation was made by the combination of a constant $\left(W_{\text {ti: }}\right)$ and a sinusoidal $\left(V_{\text {ac }}\right)$ component of voltage applied between $\mathrm{Al}$ and $\mathrm{TCO}$ electrodes. Positive and negative polarities of voltage refer to the top Al electrode being positively and negatively biased with respect to the bottom TCO electrode. The modulation frequency was fixed at $1 \mathrm{kHz}$. Here, the efrect of current through the MQW is negligible because of the large resistivity perpendicular to the layers, being more than $10^{12} \Omega \mathrm{cm}$. The broadband light with an intensity $1 \mathrm{~mW} / \mathrm{cm}^{2}$ was directed onto the transparent substrate, and the reflected light was dispersed by a monochromator. The reflection $R$ and its modulated component $\Delta R$ were detected by a photomultiplier and a lock-in amplifier. The change in transmission of the probe light through the MQW after two passes involving a reflection at the Al electrode makes a dominant contribution to the observed $\Delta R$, as indicated by Nonomura er al. ${ }^{12}$ The signal defined as a form $-\Delta R / R$ is, therefore, proportional to the field-induced change in absorntion coefficient, so far as the spectral region in the vicinity of the absorption edge is concerned.

We will discuss here the EA spectrum expected in the spectral region around the absorption edge, in which the transition between the ground subbands dominates the spectral behavior. It would be reasonable to start with Tauc's model for optical absorption, "because it leads to quantitative predictions as simple formulae. The absorption coefficient $\alpha$ in the photon energy region below the onset of the second subband transition is approximately represented by

$$
h \omega \alpha=A_{1}\left(h \omega-E_{1}\right) U\left(h \omega-E_{1}\right) \text {, }
$$

within the context of the model assuming a relaxed selection rule for wave vector $k$ in the direction parallel to the $Q W$ layer as well as a constant momentum matrix elenent for the transition. ${ }^{5}$ Here, ho denotes photon energy, $E_{\xi}$ is the energy 
separation between ground bound states in the conduction band and valence band, and $U(E)$ is a step function. $A_{1}$ is a constant proportional to the squared overlap integral of an electron and a hole wave function for the confined states.

The external electric fieid produces variations in the quantities $E_{1}$ and $A_{1}$ as follows. In the small feld limit, a second-order perturbation approach can be successfully applied, ${ }^{4}$ yiclding

$$
E_{1}(\Delta F) \cong E_{1}(0)-\left|\frac{\partial^{2} E_{1} / \partial F^{2}}{2}\right| \Delta F^{2}
$$

where $\Delta F$ denotes the field perpendicular to the $Q W$ layer. Since the electrostatic potential across the QW layer has an odd parity in the confinement direction, a first-order correction term in the perturbation expansion vanishes and does not appear in Eq. (2). The relation shows that the onset energy of the ground subband transition experiences a red shift of an extent proportional to $\Delta F^{2}$. The quantity $A_{1}$ representing the squared overiap integral also follows:

$$
A_{1}(\Delta F) \cong A_{1}(0)-\left|\frac{\partial^{2} A_{1} / \partial F^{2}}{2}\right| \Delta F^{2}
$$

The decrease of $A_{1}$ is understood as a natural consequence of the spatial separation of wave functions for electron and hole. The expressions (2) and (3) stand for a low-field limit of the QCFK effect. Field-induced change in absorption spectrum $\Delta a$ is expressed by using Eqs. (1)-(3) as

$$
\begin{aligned}
\hbar \omega \Delta \alpha= & \left(A_{l}\left|\frac{\partial^{2} E_{1} / \partial F^{2}}{2}\right|-\left|\frac{\partial^{2} A_{1} / \partial F^{2}}{2}\right|\left(\hbar \omega-E_{1}\right)\right) \\
& \times U\left(\hbar \omega-E_{l}\right) \Delta F^{2} .
\end{aligned}
$$

The important implications of $\mathrm{Eq}$. (4) are that the EA spectrum of the QW has a triangular shape, sharply rising at $\hbar \omega=E_{\text {i }}$ and then decreasing with photon energy, while the magnitude of the signal is proportional to a square of the field strength. In arriving at $\mathrm{Eq}$. (4), we neglect the effect of forbidden subband transitions enhanced due to a broken symmetry under the field. It will produce additional EA structures at each forbiden transition energy. Their contributions are, however, considered to be less pronounced compared with that associated with the first allowed transition which produces the triangular line shape.

Figure 1 presents the EA spectrum of the MQW structure $\left(L_{W}=20 \AA, L_{B}=100 \AA\right.$, and $\left.M=150\right)$. For comparison, the $T A$ spectrum ${ }^{5}$ measured on the identical MQW structure directly formed on a glass substrate is also shown in this figure. These spectral shapes of $Q W$ structure are clearly different from those commonly observed on thick film. ${ }^{5,8,9}$ As discussed in our previous paper, the step-like TA spectrum is ascribed to the subband transition, and the energy position of the step edge is identified as the onset energy. ${ }^{5}$ On the other hand, the EA spectrum exhibits a triangular line shape, which is in agreement with that expected from Eq. (4). The EA signal rapidly decaying towards higher photon energy is partly attributed to the effect of increase in absorption coeficient. The spectral structure is identified as due to the subband transition from a good coincidence of the EA peak and the TA step edge. The verticai bar in the figure indicates the position of the calculated onser encrgy of the

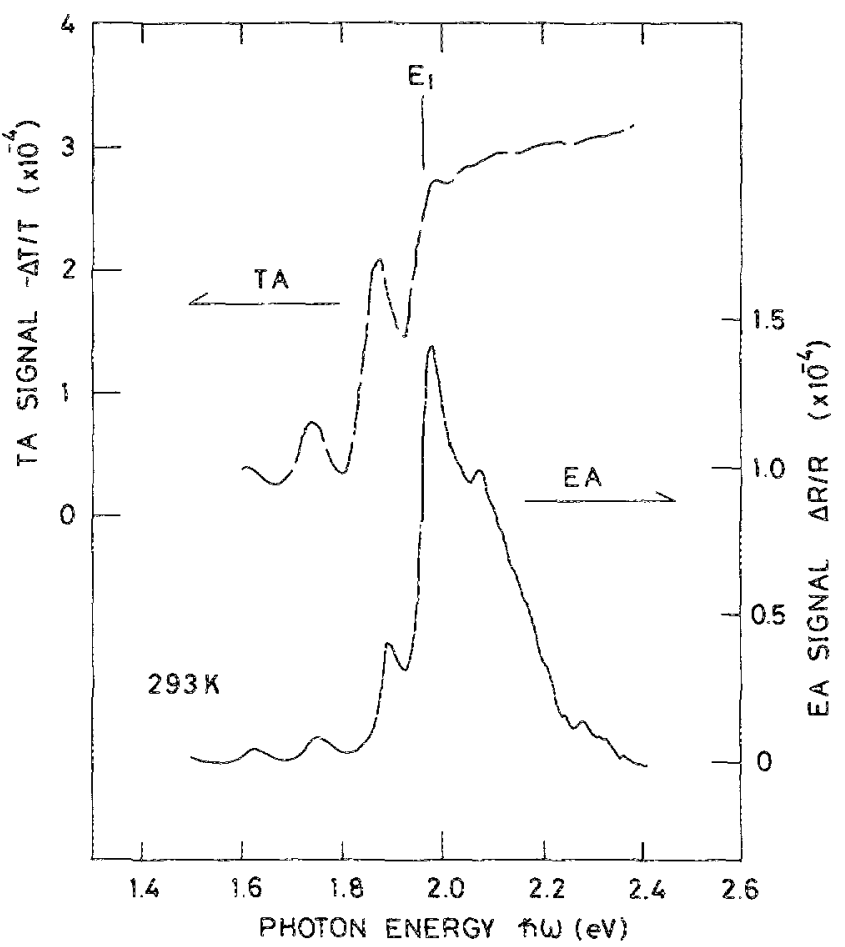

FIG. 1. TA and EA spectra for $a$-Si:H/a-SiC:H MQW structures with $L_{H}$ $=20 \AA, L_{B}=100 \AA$, and $M=150$ at temperature $293 \mathrm{~K}$. The EA spectrum was measured at $V_{\mathrm{d}}-0 \mathrm{~V}$ and $V_{\mathrm{w}}=10 \mathrm{~V}$. The vertical bar indicates the transition energy between the firsi subbands $E_{\text {l }}$ calculated from a squa1e QW model.

first subband transition $E_{1}$, which agrees well with the energy positions of the TA step-edge and the EA peak. Here, the effective masses for electron and hole were assumed to be 0.3 $m_{0}$ and $m_{0}$, respectively, in both sublayer regions, where $m_{0}$ denotes free-electron mass. These values are identical with those estimated from our recent work on differential absorption spectroscopies of $a$-Si:H-based QW structures. ${ }^{5,6}$

Roxio et $a l$. have recently studied the EA spectra of $a$ $\operatorname{Si}: \mathrm{H}\left(E_{0}=1.77 \mathrm{eV}\right) / \alpha-\operatorname{SiC} \mathrm{H}\left(E_{0}=2.22 \mathrm{eV}\right)$ superlattice structures. ${ }^{13}$ The spectral behaviors did not exhibit any features distinguishable from that of bulk materials in contrast to the present result. The disagreement may result from the difference in sample structures used in each experiment. In the combination of well and barrier materias adopted by Roxlo et al., the discontinuity in the valence band is close to zero, so that the QW effect for holes is negligible. In addition, since the widths of the well and barrier layer are set equal to each other, QW levels in the conduction band are broadened with decreasing the sublayer width due to the tunnel coupling. These effects would explain why their EA spectra exhibit no definite features ascribed to $\mathrm{QW}$ effects.

Figure 2 shows the EA signa intensity as a function of dc bias voltage $V_{\text {ac }}$ for the MOW sample $\left(L_{W}=20 \AA, L_{R}\right.$ $=60 \AA$, and $M=40$ ). Here, the modulation voltage $V_{\text {ac }}$ was held at $2 \mathrm{~V}$. The spectra measured at $V_{\text {dc }}=-8,0$, and $8 \mathrm{~V}$ are given in the inse The spectra are found to have an expected triangular shape, when one neglects bumps seen around 1.5 and $2 \mathrm{eV}$ due to interference effect. The presented data for signal intensity are nomalized by that at $0 \mathrm{~V}$, and the relative values fall within the indicated error bar over the 


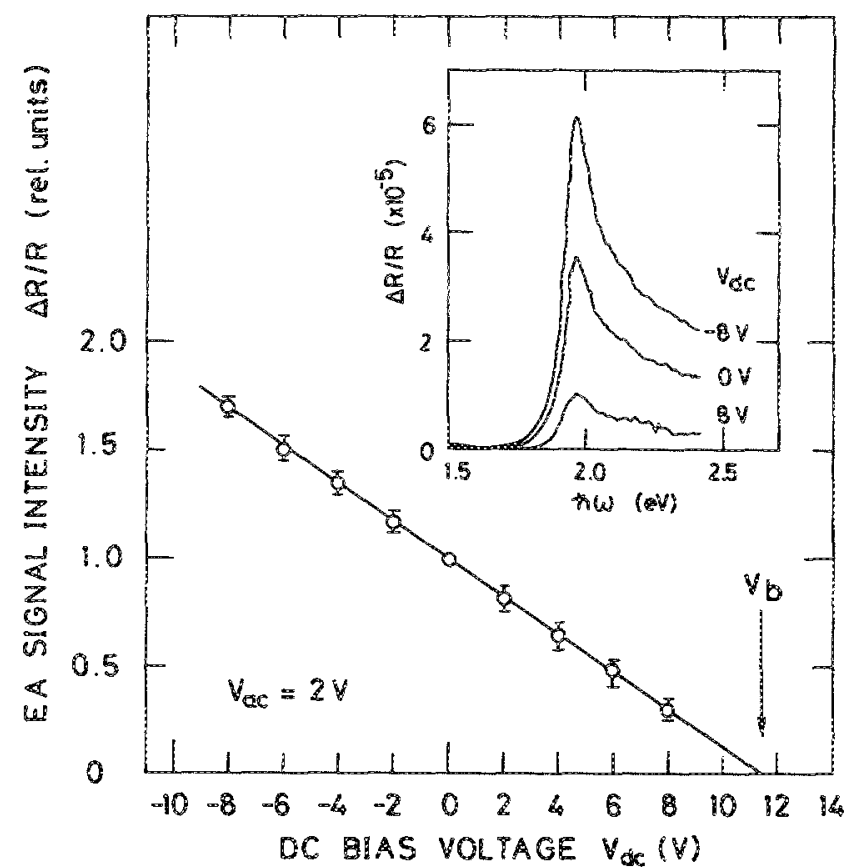

FIG. 2. EA signal intensity measured on $a-S i: H / a-S i C$ H MOW structure with $L_{W r}=20 \AA, L_{B}=60, \AA$, and $M=40$ as a function of dc bias voltage $V_{\text {w }}$. The modulation voltage $V_{\text {ac }}$ was held at $2 \mathrm{~V}$. The inset shows the EA spectra for $V_{\mathrm{d}}=-8,0$, and $8 \mathrm{~V}$.

photon energy region of experiment. Since the EA signal was obtained at the first harmonics of the modulation frequency, the quadratic field-strength dependence leads to a linear relation between the signal intensity and the dic field strength, which is the sum of the applied and builtain fields, so far as the ac field strength is kept constant. ${ }^{12,24}$ The excellent linearity found between the EA intensity and the de bias voltage ensures us that the built-in fieid is almost independent on the applied dc field. The intercept of the extrapolated line $\left(V_{b}\right)$, thereby, corresponds to the dc bias voltage required to cancel out the built-in potential drop $\phi$ across each well layer. Simple electrostatics gives the relation between $\phi$ and $V_{b}$ : $\phi=V_{b}\left[M+(M+1) \epsilon_{W} L_{B} / \epsilon_{B} L_{W}\right]^{-1}$, where $\epsilon_{W}$ and $\epsilon_{B}$ are the dielectric constants for well and barrier materials $\left(\epsilon_{W}=12\right.$ and $\left.\epsilon_{b}=6.3\right)$. Applying the equation to the experimental data in Fig. 2 yields an estimate of the built-in potential $\phi$ at $42 \mathrm{mV}$. The positive $\phi$ designates a field which points away from the substrate. Assuming that the field is distributed uniformly across the layer, the field strength is measured at $2 \times 10^{5} \mathrm{~V} / \mathrm{cm}$. An almost identical value was obtained for the sample as in Fig. 1.

Since the estimated built-in field $2 \times 10^{5} \mathrm{~V} / \mathrm{cm}$ is rather large, we have to reexamine the applicability of perturbation theory starting from a square QW model. For this purpose, we have made a numerical calculation of tunneling transmission coefficient through QW structure in the presence of electric field using a transfer matrix technique. ${ }^{15}$ The energies of transmission resonance give the positions of the energy levels within the well. ${ }^{3,4}$ The results for a $\mathrm{QW}$ of $20 \AA$ thickness indicate that the first energy levels for electron and hole both shift downward, with an extent being proportional to the square of electric field $F^{2}$ up to around $F=6 \times 10^{5} \mathrm{~V} /$ cm. Based upon perturbation theory, the quadratic field dependence was applied to the analysis of the EA signat inten- sity with varying electric field in a range less than $4 \times 10^{5}$ $\mathrm{V} / \mathrm{cm}$, as described before. The approach is therefore justified from the numerical result. The calculation also shows that the shift of the transition energy between the first subbands is about $8 \mathrm{meV}$ at $F=4 \times 10^{5} \mathrm{~V} / \mathrm{cm}$. The shift of such a small extent is likely to be buried in the width of the observed EA spectrum peak, and is considered to be insuffcient to be observed in this experimental condition. Therefore, the unperturbed square $Q W$ model is a reasonable approximation for the interpretation of the subband transition energy.

The built-in field might be attributed to asymmetrically placed interfacial defects with charge, as suggested by Roxlo et al. ${ }^{14}$ Following this picture, the interface defect density is estimated to be about $10^{12} \mathrm{~cm}{ }^{2}$ for the present QW system, which is of the similar order as in $a-\mathrm{Si}: \mathrm{H} / a-\mathrm{SiN}$ :H multilayered structures. ${ }^{14}$ The extent is larger by one order of magnitude than that of a latice-matched crystalline superlatice system of GaAs and $\mathrm{Ga}_{0.7} \mathrm{Al} \mathrm{l}_{0.3} \mathrm{As}$. ${ }^{6}$ The presence of defects suggests the increased structural disorder in the interface region, which might work as another source of broadening in energy levels within the $\mathrm{QW}$, in addition to the broadening caused by inherent disorder in the material.

In summary, EA spectrum has been investigated on $a$ Si:H/a-SiC:H MQW structures. The spectrum exhibits a triangular shape. The behavior is well interpreted as arising from the field-induced modincation of the subband transition. The peak energy of EA spectrum gives the transition energy, which is in good agreement with that determined from TA experiment. The built-in potential across the $\mathrm{OW}$ layer is estimated to be $42 \mathrm{mV}$, indicating that the reflection symmetry is slightly broken in the $Q W$ potential.

'D. A. B. Miller, D. S. Chemla, and S. Schmitt-Rink, in Optical Nonlinearities and Instabilities in Semiconductors, edited by H. Haug (Academic, Orlando, 1988), pp. 325-359.

D. A. B. Miller, D.S. Chemla, and S. Schmitt-Rink, Phys. Key. B33, 6976 (1986).

'D. A. B. Miller, D. S. Chemla, T. C. Damen, A. C. Gossard, W. Wiegmann, T. H. Wood, and C. A. Burrus, Phys. Rev. B 32, 1043 (1985).

"G. Bastard, in Interfaces, Quantum Wells, and Superlattices, edited by C.

R. Leavens and R. Taylor (Plenum, New York and London, 1988), pp. 189-209.

${ }^{5}$ K. Hattori, T. Mori, H. Okamoto, and Y. Hamakawa, Phys. Rev. Lett. 68 , 825 (1988).

'K. Hattor, T. Mori, H. Okamoto, and Y. Hamakawa, Appl. "hys. Lett. 53, 2170 (1988).

${ }^{7}$ S. Miyazaki, Y. Thara, and M. Mirose, Phys. Rev. Lett. 59, 125 (1987).

${ }^{8}$ Y. Hamakawa, in Semiconducrors and Semimetals, edited by I. I. Pankove (Academic, New York, 1984), Vol. 21, Part B, pp. 14i-158.

${ }^{9} \mathrm{G}$. Weiser, U. Dersch, and P. Thomas, Philos. Mag. B 57, 721 (1988).

"For example, A. Frova and A. Selloni, in Tetrahedrally-londed Amorphous Semiconductors, edited by D. Adler and $\mathrm{H}$. Fritzsche (Plenum, New York and London, 1985), pp. 271-285.

"K. Ilattori, T. Mori, Il. Okamoto, and Y. Hamakawa, Advances in Disordered Semiconduciors Wol. 1, Amorphous Silicon and Related Materials, edited by H. Fritzsche (World Scientific, Singapore, 1988), pp. 957-976.

"S. Nonomura, H. Okamoro, and Y. Hamakawa, Appl. Phys. A 32, 31 (1983).

C. B. Roxlo, B. Abeles, and P. D. Persans, Appl. Phys. Letr. 45, 1132 (1984).

${ }^{14} \mathrm{C}$. B. Roxlo, B. Abeles, and T. Tiedje, Phys. Rev. Lett. 52, 1994 (1984). 'M. O. Vassell, J. Lee, and I. F. Lockwood, I. Appl. Phys. 54, 5206 (1983).

in A. Manger, S. I. Feng, and J. C. Bourgoin, Appl. Phys. Leti. 51, 27 (1987). 